University of New Orleans

ScholarWorks@UNO

$11-20-2011$

\title{
Circular and near-circular polarization states of evanescent monochromatic light fields in total internal reflection
}

R. M. A Azzam

University of New Orleans, razzam@uno.edu

Follow this and additional works at: https://scholarworks.uno.edu/ee_facpubs

Part of the Electrical and Electronics Commons, and the Optics Commons

\section{Recommended Citation}

R. M. A. Azzam, "Circular and near-circular polarization states of evanescent monochromatic light fields in total internal reflection," Appl. Opt. 50, 6272-6276 (2011).

This Article is brought to you for free and open access by the Department of Electrical Engineering at ScholarWorks@UNO. It has been accepted for inclusion in Electrical Engineering Faculty Publications by an authorized administrator of ScholarWorks@UNO. For more information, please contact scholarworks@uno.edu. 


\title{
Circular and near-circular polarization states of evanescent monochromatic light fields in total internal reflection
}

\author{
R. M. A. Azzam \\ Department of Electrical Engineering, University of New Orleans, New Orleans, \\ Louisiana 70148, USA (razzam@uno.edu) \\ Received 12 August 2011; accepted 19 September 2011; \\ posted 4 October 2011 (Doc. ID 152799); published 18 November 2011
}

\begin{abstract}
Conditions for the production of near-circular polarization states of the evanescent field present in the rarer medium in total internal reflection of incident monochromatic $p$-polarized light at a dielectricdielectric planar interface are determined. Such conditions are satisfied if high-index (>3.2) transparent prism materials (e.g., GaP and $\mathrm{Ge}$ ) are used at angles of incidence well above the critical angle but sufficiently below grazing incidence. Furthermore, elliptical polarization of incident light with nonzero $p$ and $s$ components can be tailored to cause circular polarization of the resultant tangential electric field in the plane of the interface or circular polarization of the transverse electric field in a plane normal to the direction of propagation of the evanescent wave. Such polarization control of the evanescent field is significant, e.g., in the fluorescent excitation of molecules adsorbed at solid-liquid and solid-gas interfaces by total internal reflection. (C) 2011 Optical Society of America

OCIS codes: $\quad 240.0240,240.6690,260.0260,260.5430,260.6970$.
\end{abstract}

\section{Introduction}

Total internal reflection (TIR) of an incident monochromatic plane wave of arbitrary polarization state (with nonzero $p$ and $s$ components present) at the planar interface between two transparent media generates an evanescent field in the rarer medium whose state of polarization is in general elliptical and three-dimensional [1-4]. In this paper, we consider conditions that lead to circular or near-circular polarization of the evanescent refracted wave. The transparent media of incidence and refraction of refractive indices $n_{0}$ and $n_{1}$ are assumed to be linear, homogeneous, optically isotropic, and nonmagnetic. The $e^{j \omega t}$ time dependence, Nebraska-Muller conventions $[\underline{5}, 6]$, and the $x y z$ coordinate system shown in Fig. 1 are adopted.

The $3 \times 1$ generalized Jones vectors of the incident $(i)$ and evanescent $(e)$ refracted waves are written as

0003-6935/11/336272-05\$15.00/0

(C) 2011 Optical Society of America

$$
\begin{gathered}
\boldsymbol{E}_{i}=\left[\begin{array}{lll}
E_{i x} & E_{i y} & E_{i z}
\end{array}\right]^{t} \\
=\left[\begin{array}{lll}
(\sin \phi) E_{i p} & E_{i s} & (-\cos \phi) E_{i p}
\end{array}\right]^{t}, \\
\boldsymbol{E}_{e}=\left[\begin{array}{lll}
E_{e x} & E_{e y} & E_{e z}
\end{array}\right]^{t},
\end{gathered}
$$

where $t$ indicates the transpose. In Eq. (1), $E_{i p}$ and $E_{i s}$ are phasors that represent the time-harmonic $p$ and $s$ components of the incident electric field and $\phi$ is the angle of incidence that exceeds the critical angle of TIR, $\phi>\phi_{c}=\sin ^{-1}\left(n_{1} / n_{0}\right)$. Application of boundary conditions to the normal $(x)$ and tangential ( $y$ and $z$ ) components of the electric field at the dielectricdielectric interface leads to the following relations between the Cartesian components of the evanescent and incident fields:

$$
E_{e x}=T_{11} E_{i x}, \quad E_{e y}=T_{22} E_{i y}, \quad E_{e z}=T_{33} E_{i z}
$$




$$
\begin{aligned}
& T_{11}=N^{2}\left(1+r_{p}\right), \\
& T_{22}=\left(1+r_{s}\right), \\
& T_{33}=\left(1-r_{p}\right) .
\end{aligned}
$$

In Eqs. (4), $N=n_{0} / n_{1}$ is the high-to-low refractive index ratio of the two dielectrics, and $r_{p}, r_{s}$ are the TIR interface Fresnel coefficients that are pure phase factors:

$$
r_{p}=\exp \left(j \delta_{p}\right), \quad r_{s}=\exp \left(j \delta_{s}\right) .
$$

The TIR phase shifts $\delta_{p}, \delta_{s}$ are functions of $N, \phi$ given by [7]

$$
\begin{gathered}
\tan \left(\delta_{p} / 2\right)=N \sec \phi\left(N^{2} \sin ^{2} \phi-1\right)^{1 / 2} \\
\tan \left(\delta_{s} / 2\right)=N^{-1} \sec \phi\left(N^{2} \sin ^{2} \phi-1\right)^{1 / 2} .
\end{gathered}
$$

The rest of the paper proceeds as follows: In Section 2 , incident $p$-polarized light $\left(E_{i s}=0\right)$ is assumed; such light produces elliptical polarization of the evanescent refracted field in the $x z$ plane of incidence (plane of the page in Fig. 1) [1,8]. We show that near-circular polarization is achieved at sufficiently large but realistic values of $N, \phi$. In Section 3 , the incident polarization state $\chi_{i}=E_{i s} / E_{i p}$ that causes circular polarization of the resultant of the $y$ and $z$ tangential components of the electric field in the plane boundary is determined. In Section $\underline{4}$, the incident polarization state $\chi_{i}=E_{i s} / E_{i p}$ that causes circular polarization of the combined $x$ and $y$ transverse components of the electric field of the evanescent wave propagating in the $z$ direction is also obtained. Polarization control of the evanescent field is important in studies of fluorescent excitation of molecules adsorbed at solid-liquid and solid-gas interfaces using TIR [8-10]. Finally, Section $\underline{5}$ gives a brief summary of this work.

\section{Near-Circular Polarization of the Evanescent Field Generated by Incident $p$-Polarized Light}

The state of polarization of the evanescent refracted field generated by incident $p$-polarized $\left(E_{i s}=0\right)$ light is defined by the complex ratio []

$$
\chi_{e}=E_{e z} / E_{e x} .
$$

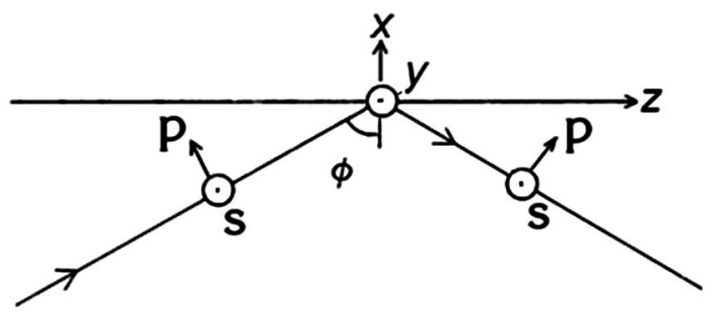

Fig. 1. Total internal reflection of $p$ - and $s$-polarized light at a dielectric-dielectric interface at angle of incidence $\phi$ and the reference $x y z$ coordinate system used to describe the incident and evanescent refracted wave fields.

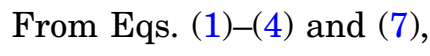

$$
\chi_{e}=\left(\cot \phi / N^{2}\right)\left[\left(r_{p}-1\right) /\left(r_{p}+1\right)\right]
$$

is obtained. With $r_{p}=\exp \left(j \delta_{p}\right)$, Eq. ( $\left.\underline{8}\right)$ simplifies to

$$
\chi_{e}=\left(\cot \phi / N^{2}\right)\left[j \tan \left(\delta_{p} / 2\right)\right] .
$$

Substitution of $\tan \left(\delta_{p} / 2\right)$ from Eqs. ( $\left.\underline{6}\right)$ in Eq. (ㅁ) leads to the simple result

$$
\chi_{e}=j b / a=j e=j\left[1-(\csc \phi / N)^{2}\right]^{1 / 2} .
$$

$\chi_{e}$ of Eq. (10) describes an ellipse of polarization with semimajor and semiminor axes $a$ and $b$ aligned with the $x$ and $z$ axes, respectively, and with ellipticity $e=b / a$. In Appendix A we provide an alternate derivation of Eq. (10), which is based on Gauss's law $\nabla \cdot \vec{E}_{e}=0$.

Near-circular polarization is achieved if $e=b / a \geq$ 0.95 . For illustration, Fig. 2 shows a circular state (CS) and an elliptical near-circular state (ENCS) that correspond to $e=1$ and $e=0.95$, respectively.

Figure $\underline{3}$ shows $e=b / a$ of Eq. (10) plotted as a function of $\phi$ from the critical angle $\phi=\phi_{c}=$ $\arcsin (1 / N)$ to grazing incidence $\phi=90^{\circ}$ at constant values of the index ratio $N$ from 2 to 6 in steps of 1 . Note that $e=0$, which represents linear polarization in the $x$ direction, occurs at the critical angle $\phi_{c}$ and that $e$ asymptotically reaches maximum saturation value

$$
e_{\max }=\left[1-(1 / N)^{2}\right]^{1 / 2}=\cos \phi_{c}
$$

at $\phi=90^{\circ}$. The value of $N$ required to achieve a certain $e_{\max }$ is readily obtained from Eq. (11) as

$$
N=1 / \sqrt{1-e_{\max }^{2}}
$$

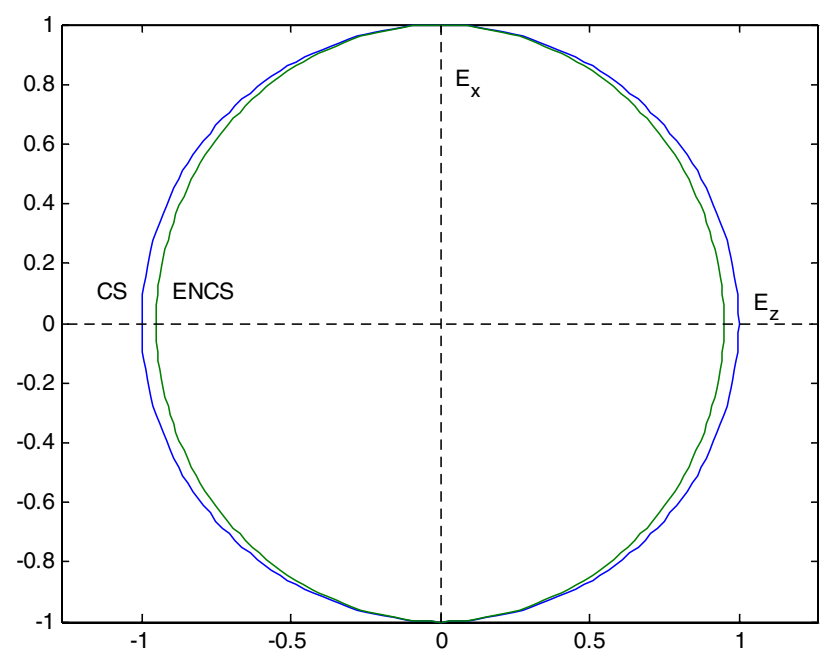

Fig. 2. (Color online) Circular polarization state (CS, $e=1)$ and an elliptical near-circular state (ENCS) with $e=b / a=0.95$. 


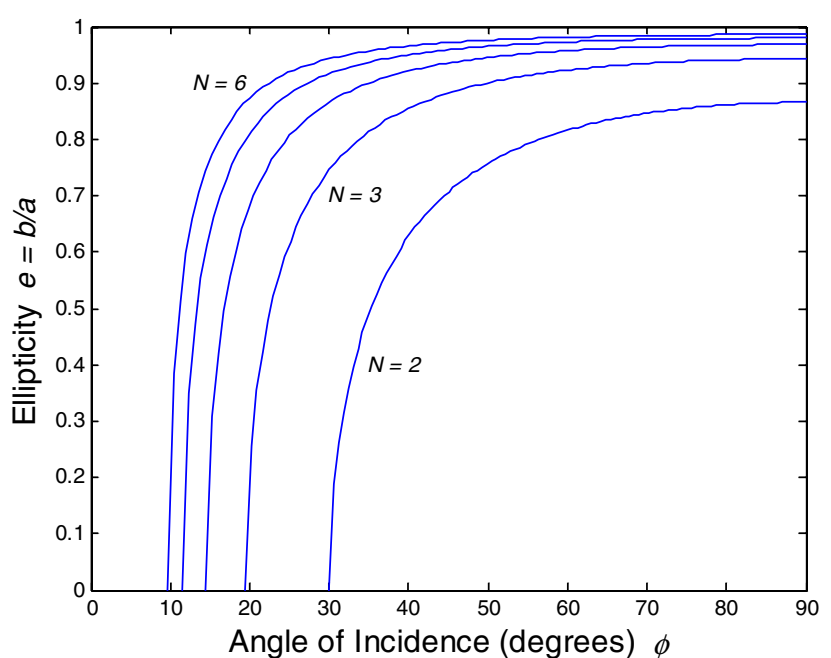

Fig. 3. (Color online) Ellipticity $e=b / a$ of the evanescent electric field [Eq. (10)] is plotted as a function of $\phi$ from the critical angle to grazing incidence at constant values of $N$ from 2 to 6 in steps of 1 .

Equation (12) indicates that $e_{\max } \geq 0.95$ is achieved if $N \geq 3.2026$.

As a specific example consider TIR at the GaP-air interface. $\mathrm{GaP}$ is transparent in the $620-760 \mathrm{~nm}$ red visible spectrum and also in the near- and middle-IR and has a refractive index of 3.3 at the $\mathrm{He}-\mathrm{Ne}$ laser wavelength $\lambda=633 \mathrm{~nm}$ [11]. With $N=3.3$, Eq. (11) gives $e_{\max }=0.953$. And according to Eq. (10), $e$ increases slightly from 0.950 at $\phi=76.04^{\circ}$ to $e_{\max }=$ 0.953 at $\phi=90^{\circ}$. This confirms that ENCSs can be achieved in TIR at the GaP-air interface at incidence angles sufficiently below $90^{\circ}$.

As another example, consider TIR of IR radiation at the Ge-air interface for which $N \approx 4$ over a broad bandwidth [12]. Substitution of $N=4$ in Eq. (11) gives $e_{\max }=\sqrt{15 / 16}=0.968$. Equation (10) indicates that $e$ increases from 0.950 at $\phi=\overline{53} .19^{\circ}$ to $e_{\max }=0.968$ at $\phi=90^{\circ}$. Once again this proves that ENCSs are realizable in TIR of IR radiation at the Ge-air interface at incidence angles well below $90^{\circ}$.

\section{Circular Polarization of the Tangential Electric Field of the Evanescent Wave in the Plane of the Interface}

For TIR of incident monochromatic light of arbitrary polarization,

$$
\chi_{i}=E_{i s} / E_{i p},
$$

the polarization state of the tangential $(t)$ electric field of the evanescent wave in the plane of the interface is given by

$$
\chi_{e t}=E_{e z} / E_{e y} .
$$

From Eqs. (1)-(ㅁ), (13), and (14) we obtain

$$
\chi_{e t}=\left(\cos \phi / \chi_{i}\right)\left[\left(r_{p}-1\right) /\left(r_{s}+1\right)\right] .
$$

Circular polarization of the tangential field is achieved if

$$
\chi_{e t}= \pm j
$$

We take $\chi_{e t}=+j$ and use Eqs. (ㅁ), (13), and (15) to obtain

$$
\begin{aligned}
\chi_{i} & =E_{i s} / E_{i p} \\
& =\cos \phi\left[\sin \left(\delta_{p} / 2\right) / \cos \left(\delta_{s} / 2\right)\right] \exp \left[j\left(\delta_{p}-\delta_{s}\right) / 2\right] .
\end{aligned}
$$

Use of $\chi_{e t}=-j$ instead of $\chi_{e t}=+j$ simply changes the phase angle of the last term of Eq. (17) by $\pi$.

Equation (17) specifies the incident elliptical polarization state that causes the tangential electric field of the evanescent wave in the $y z$ plane to be circularly polarized. From Eqs. (6) and (17) the required ratio of amplitudes of the $s$ and $p$ components of incident light is given by

$$
\begin{aligned}
\left|\chi_{i}\right| & =\left|E_{i s}\right| /\left|E_{i p}\right| \\
& =\left(N^{2} \sin ^{2} \phi-1\right)^{1 / 2} /\left[\left(N^{2}+1\right) \sin ^{2} \phi-1\right]^{1 / 2},
\end{aligned}
$$

and the associated phase difference is

$$
\delta_{i s}-\delta_{i p}=\arctan \left[\left(N^{2} \sin ^{2} \phi-1\right)^{1 / 2} /(N \sin \phi \tan \phi)\right] .
$$

From Eq. (18) it is apparent that $\left|\chi_{i}\right|<1$. Figure 4 shows $\left|\chi_{i}\right|$ plotted as a function of $\phi$ from the critical angle $\phi=\phi_{c}=\arcsin (1 / N)$ to grazing incidence $\phi=90^{\circ}$ for constant values of $N$ from 2 to 6 in steps of 1 . Notice that $\left|\chi_{i}\right|$ initially rises steeply just above the critical angle and saturates at larger angles as

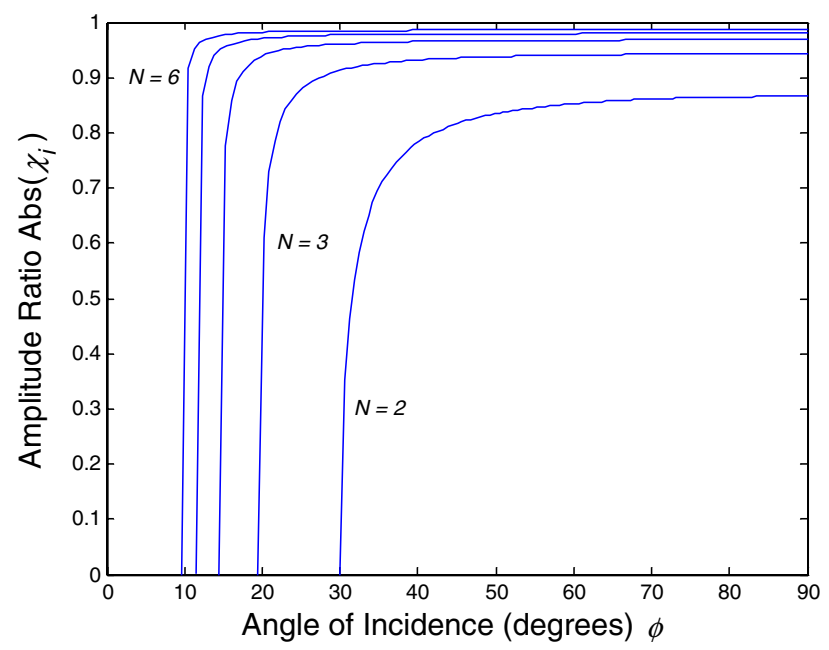

Fig. 4. (Color online) Amplitude ratio $\left|\chi_{i}\right|=E_{i s} / E_{i p}$ [Eq. (18)] required to produce circular polarization of the tangential electric field in the plane of the interface is plotted as a function of $\phi$ from the critical angle to grazing incidence for constant values of $N$ from 2 to 6 in steps of 1 . 
$\phi \rightarrow 90^{\circ}$. From Eq. (18) the saturation value of $\left|\chi_{i}\right|$ at $\phi=90^{\circ}$ is given by

$$
\left|\chi_{i}\right|_{90}=\left(N^{2}-1\right)^{1 / 2} / N
$$

Also of interest is $\left|\chi_{i}\right|$ at the angle of incidence $\phi_{m}=\arcsin \sqrt{2 /\left(N^{2}+1\right)}$ at which the TIR differential phase shift $\left(\delta_{p}-\delta_{s}\right)$ is maximum [7]. At $\phi=\phi_{m}$, Eq. (18) simplifies to

$$
\left|\chi_{i}\right|=\left(N^{2}-1\right)^{1 / 2} /\left(N^{2}+1\right)^{1 / 2} .
$$

Figure 5 shows the amplitude ratios given by Eqs. (20) and (21) plotted versus $N$ for $2 \leq N \leq 6$.

In Fig. $\underline{6}$, the phase difference between the incident $s$ and $p$ components at which the tangential electric field becomes circularly polarized [Eq. (19)] is plotted as a function of $\phi$ from the critical angle to grazing incidence for constant values of $N$ from 2 to 6 in steps of 1 .

Figures $\underline{4}$ and $\underline{6}$ indicate that to produce circular polarization $\left(\chi_{e t}=+j\right)$ of the tangential electric field in the plane of the boundary the incident polarization $\chi_{i}$ is limited to the first quadrant of the unit circle in the complex plane. A polarization state generator, e.g., a combination of a linear polarizer and a quarter-wave retarder, is used to polarize the incident light in any desired state [6] .

\section{Circular Polarization of the Transverse Electric Field of the Evanescent Wave}

The polarization of the transverse $(T)$ electric field of the $z$-traveling evanescent wave in the $x y$ plane is given by

$$
\chi_{e T}=E_{e y} / E_{e x}
$$

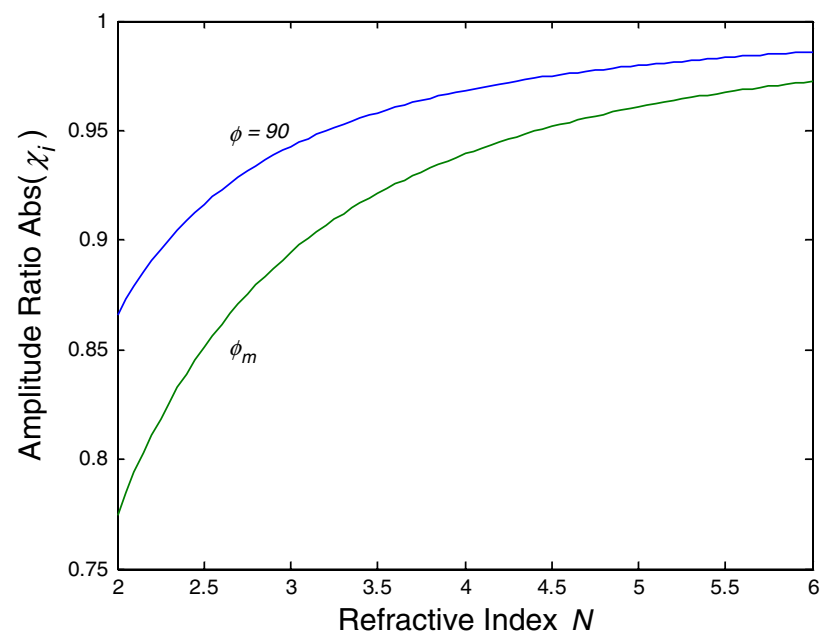

Fig. 5. (Color online) Amplitude ratio $\left|\chi_{i}\right|=E_{i s} / E_{i p}$ for circular polarization of the tangential electric field at $\phi=90^{\circ}$ and $\phi_{m}=$ $\arcsin \sqrt{2 /\left(N^{2}+1\right)}$ [Eqs. (20) and (21)] is plotted versus $N$ in the range $2 \leq N \leq 6$.

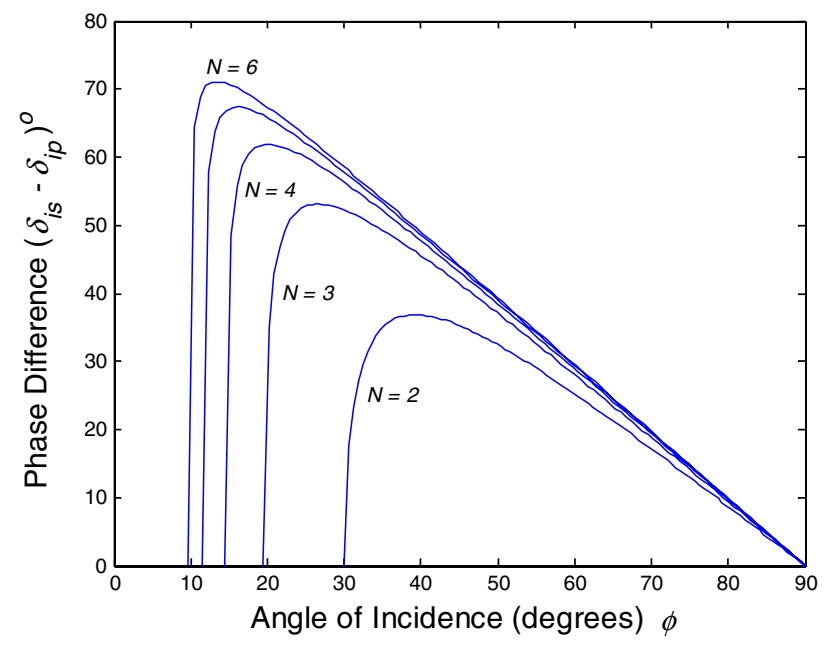

Fig. 6. (Color online) Phase difference $\delta_{i s}-\delta_{i p}$ [Eq. (19)] between the incident $s$ and $p$ components of incident light, which is required to produce circular polarization of the tangential electric field, is plotted as a function of $\phi$ from the critical angle to grazing incidence for constant $N$ values from 2 to 6 in steps of 1 .

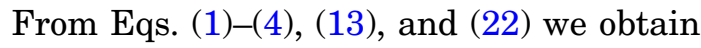

$$
\chi_{i}=\chi_{e T}\left(N^{2} \sin \phi\right)\left[\left(r_{p}+1\right) /\left(r_{s}+1\right)\right] .
$$

Circular polarization of the transverse field is achieved when $\chi_{e T}= \pm j$. We take $\chi_{e T}=+j$ and follow steps similar to those used in Section $\underline{3}$ to obtain

$$
\left|\chi_{i}\right|=\left|E_{i s}\right| /\left|E_{i p}\right|=(N \sin \phi) /\left[\left(N^{2}+1\right) \sin ^{2} \phi-1\right]^{1 / 2},
$$

$$
\begin{aligned}
\delta_{i s}-\delta_{i p}= & \arctan \left[\left(N^{2} \sin ^{2} \phi-1\right)^{1 / 2} /(N \sin \phi \tan \phi)\right] \\
& +\pi / 2 .
\end{aligned}
$$

If $\chi_{e T}=-j$ is selected instead of $\chi_{e T}=+j$ the rightmost term of Eq. (25) changes from $+\pi / 2$ to $-\pi / 2$. Equation (25) differs from Eq. (19) only in the $+\pi / 2$ added term.

Figure 7 shows $\left|\chi_{i}\right|$ of Eq. (24) plotted as a function of $\phi$ from the critical angle to grazing incidence at constant values of $N$ from 2 to 6 in steps of 1 . Note that $\left|\chi_{i}\right|>1$ for all values of $N$ and $\phi$ and that the limiting values of $\left|\chi_{i}\right|$ at $\phi_{c}$ and $90^{\circ}$ are $N$ and 1 , respectively. For $\chi_{e T}=+j, \chi_{i}$ occupies the region of the second quadrant of the complex plane outside the unit circle.

\section{Summary}

Near-circular polarization of the evanescent field present in the rarer medium under conditions of TIR of incident $p$-polarized light at a dielectricdielectric planar interface is achieved with highindex (>3.2) transparent prism materials (e.g., $\mathrm{GaP}$ and $\mathrm{Ge}$ ) and at angles of incidence well above the critical angle but sufficiently below grazing incidence. Elliptical polarization of incident light with 


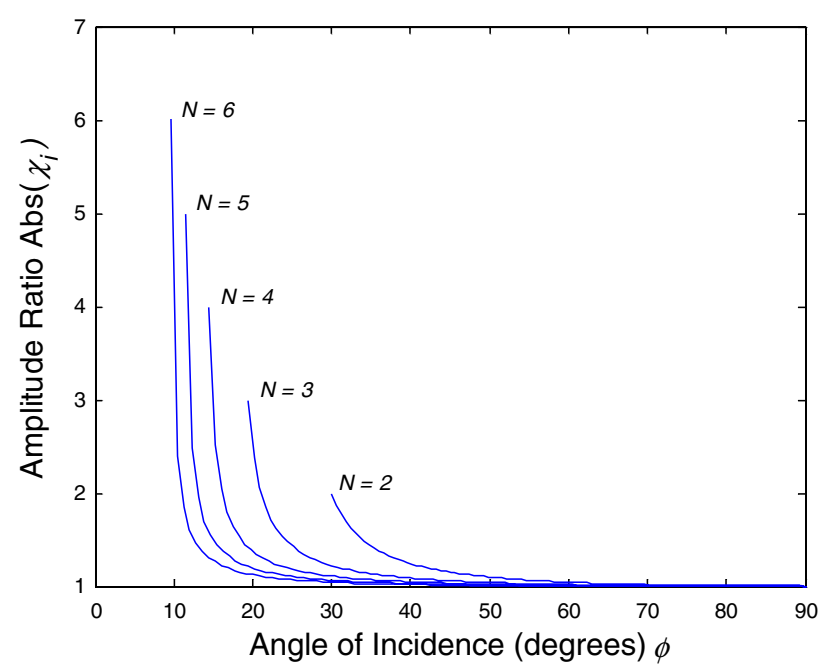

Fig. 7. (Color online) Amplitude ratio $\left|\chi_{i}\right|=E_{i s} / E_{i p}$ [Eq. (24)], which is required to produce circular polarization of the transverse electric field of the evanescent wave, is plotted as a function of $\phi$ from the critical angle to grazing incidence for $N$ values from 2 to 6 in steps of 1 .

nonzero $p$ and $s$ components can be selected to produce circular polarization of the resultant tangential electric field in the plane of the interface or circular polarization of the transverse electric field in a plane normal to the direction of propagation of the evanescent wave. Control of the polarization of the evanescent light field in TIR influences its interaction with oriented molecules adsorbed at solid-liquid and solid-gas interfaces via the dot and cross products of the optical electric field with the electric dipole moments of such molecules [13].

\section{Appendix A}

The complex wave vector of the evanescent refracted plane wave in the Cartesian coordinate system of Fig. 1 is given by

$$
\vec{k}_{e}=-j k_{e x} \hat{x}+k_{e z} \hat{z},
$$

and $\hat{x}, \hat{z}$ are unit vectors along the positive $x$ and $z$ axes. Phase matching across the boundary requires that the $z$ components of the incident and refracted wave vectors be equal, i.e.,

$$
k_{e z}=k_{i z}=\left(2 \pi n_{0} / \lambda\right) \sin \phi,
$$

where $\lambda$ is the vacuum wavelength of light. We also have

$$
\vec{k}_{e} \cdot \vec{k}_{e}=\left(2 \pi n_{1} / \lambda\right)^{2}=k_{e z}^{2}-k_{e x}^{2} .
$$

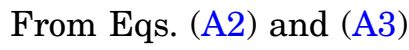

$$
k_{e x}=\left(2 \pi n_{1} / \lambda\right)\left(N^{2} \sin ^{2} \phi-1\right)^{1 / 2}
$$

is obtained, where $N=n_{0} / n_{1}$.
For incident $p$-polarized light the evanescent wave field is given by

$$
\vec{E}_{e}(x, y, z)=\left[\begin{array}{lll}
E_{e x} & 0 & E_{e z}
\end{array}\right]^{t} \exp \left(-k_{e x} x\right) \exp \left(-j k_{e z} z\right) .
$$

In the absence of free charge in the medium of refraction, the wave field of Eq. (A5) must satisfy Gauss's law

$$
\nabla \cdot \vec{E}_{e}=0
$$

Setting the divergence of the right-hand side of Eq. (A5) equal to zero leads to

$$
\chi_{e}=E_{e z} / E_{e x}=j k_{e x} / k_{e z} .
$$

If $k_{e z}$ and $k_{e x}$ of Eqs. (A2) and (4) are substituted in Eq. (A7) we obtain

$$
\chi_{e}=j\left[1-(\csc \phi / N)^{2}\right]^{1 / 2},
$$

in agreement with Eq. (10).

\section{References}

1. F. de Fornel, Evanescent Waves (Springer, 2001).

2. L. Novotny and B. Hecht, Principles of Nano-Optics (Cambridge, 2006).

3. L. Józefowski, J. Fiutowski, T. Kawalec, and H.-G. Rubahn, "Direct measurement of the evanescent-wave polarization state," J. Opt. Soc. Am. B 24, 624-628 (2007).

4. A. Norrman, T. Setälä, and A. T. Friberg, "Partial coherence and partial polarization in random evanescent fields on lossless interfaces," J. Opt. Soc. Am. A 28, 391-400 (2011).

5. R. H. Muller, "Definitions and conventions in ellipsometry," Surf. Sci. 16, 14-33 (1969).

6. R. M. A. Azzam and N. M. Bashara, Ellipsometry and Polarized Light (North-Holland, 1987).

7. R. M. A. Azzam, "Phase shifts that accompany total internal reflection at a dielectric-dielectric interface," J. Opt. Soc. Am. A 21, 1559-1563 (2004)

8. E. H. Hellen, R. M. Fulbright, and D. Axelrod, "Total internal reflection fluorescence theory and applications at biosurfaces," in Spectroscopic Membrane Probes, L. M. Loew, ed. (CRC Press, 1988), Vol. II, pp. 47-79.

9. K. H. Drexhage, "Interaction of light with monomolecular dye layers," in Progress in Optics, E. Wolf, ed. (Elsevier, 1974), Vol. 12, pp. 163-232.

10. S. E. Sund, J. A. Swanson, and D. Axelrod, "Cell membrane orientation visualized by polarized total internal reflection fluorescence," Biophys. J. 77, 2266-2283 (1999).

11. A. Borghesi and G. Guizzetti, "Gallium phosphide," in Handbook of Optical Constants of Solids, E. D. Palik, ed. (Academic, 1985), pp. 445-464.

12. W. J. Tropf, M. E. Thomas, and T. J. Harris, "Properties of crystals and glasses," in Handbook of Optics, M. Bass, ed. (McGraw-Hill, 1995), Vol. I, Chap. 33.

13. J. Michl and E. W. Thulstrup, Spectroscopy with Polarized Light (VCH, 1995). 